\title{
Maximum entropy principle for rarefied polyatomic gases
}

\author{
Milana Pavića,b, Tommaso Ruggeric ${ }^{\mathrm{c}}$, Srboljub Simićc ${ }^{\mathrm{d}, *}$ \\ ${ }^{a}$ Department of Mathematics and Informatics, Faculty of Sciences, University of Novi \\ Sad, Trg Dositeja Obradovića 4, Serbia \\ ${ }^{b}$ CMLA, ENS Cachan, PRES UniverSud Paris, 61 Avenue du Président Wilson, \\ F-94235 Cachan Cedex, France \\ ${ }^{c}$ Department of Mathematics and Research Center of Applied Mathematics \\ (C.I.R.A.M.), University of Bologna, Via Saragozza 8, 40123 Bologna, Italy \\ ${ }^{d}$ Department of Mechanics, Faculty of Technical Sciences, University of Novi Sad, Trg \\ Dositeja Obradovića 6, Serbia
}

\begin{abstract}
The aim of this paper is to show that procedure of maximum entropy principle for closure of moments equations for rarefied monatomic gases can be extended also to polyatomic gases. The main difference with respect to the usual procedure is existence of two hierarchies of macroscopic equations for moments of suitable distribution function, in which the internal energy of a molecule is taken into account. The field equations for 14 moments of distribution function, which include dynamic pressure, are derived. The entropy and the entropy flux are shown to be a generalization of the ones for classical Grad's distribution. The results are in perfect agreement with the recent macroscopic approach of extended thermodynamics for real gases.
\end{abstract}

Keywords: maximum entropy principle, kinetic theory of gases, extended thermodynamics

2000 MSC: 76P05, 82C40

\footnotetext{
*Corresponding author

Email addresses: milana.pavic@dmi.uns.ac.rs (Milana Pavić), tommaso.ruggeri@unibo.it (Tommaso Ruggeri), ssimic@uns.ac.rs (Srboljub Simić)
} 


\section{Introduction}

The aim of this paper is to establish a macroscopic model for non-equilibrium processes in rarefied polyatomic gases using the approach of maximum entropy principle (MEP). To reach that goal we use the kinetic model proposed by Bourgat, Desvillettes, Le Tallec and Perthame [1], in which the distribution function depends on an additional continuous variable that takes into account the internal energy of a molecule.

The entropy maximization, which has its roots in statistical mechanics and information theory, proved to be successful in the case of monatomic gases. Namely, it was shown (see [2]) that in the case of 13 fields the following three procedures yield the same macroscopic equations for monatomic gases: (i) Grad's moment method, (ii) extended thermodynamics (ET) of viscous, heat-conducting gases and (iii) maximization of entropy. An interesting review on irreversible thermodynamics, which also elucidates different pathways to macroscopic equations, is recently given by Müller and Weiss [3].

The closure problem for suitable set of macroscopic equations is at the heart of the issue and each of these procedures is aimed at solving it. The Grad's moment method is focused on finding the approximate non-equilibrium velocity distribution function $f(t, \mathbf{x}, \boldsymbol{\xi})$ which closes the system of balance laws in the physical case of 13 moments. The celebrated solution, given by Grad [4], is based upon expansion of distribution function in terms of tensorial Hermite polynomials. This procedure led to the system of balance laws for 13 moments of $f$, i.e. fields of mass density, velocity, temperature, stress tensor and heat flux.

The extended thermodynamics (ET) $[2,6]$, as primarily macroscopic theory, arrived at the same set of equations by imposing universal principles of relativity, entropy inequality with convex entropy. Motivated by the similarity of ET and moment equations derived from the Boltzmann equation on one hand, and Kogan's observation that Grad's distribution function maximizes the entropy [5] on the other, a maximum entropy principle was established first by Dreyer [7]. In the first edition of the book of ET of Müller and Ruggeri [8] this procedure was extended for any number of moments. Successively, similar result was given by Levermore [9]. The complete equivalence between entropy principle and MEP was proved subsequently by Boillat and Ruggeri $[10]$.

The success in the study of monatomic gases by the methods previously 
described have not been achieved in the case of polyatomic gases. The inclusion of new degrees of freedom (rotation and vibration) enriched the physical insight at the expense of mathematical coherence of the procedure [11]. In particular, internal energy density is no longer related to the trace of the second moment of distribution function, which prevents construction of simple hierarchy of transfer equations for moments. Moreover, complex physical description made solution procedures extremely complicated even for slightly involved problems.

Concerning the kinetic approach, a new light was shed on the problem when the model for binary collisions was introduced by Borgnakke and Larsen [12], which takes into account an exchange of energy (other than translational) during the collision. It was initially used for Monte Carlo simulations of polyatomic gases, but also applied for derivation of appropriate Boltzmann equation [1].

In the macroscopic framework, an ET theory for real gases has been successfully developed only very recently by Arima, Taniguchi, Ruggeri and Sugiyama [13], after several pioneering papers [14, 15]. This is the theory of 14 fields of mass density, velocity, temperature, viscous stress, dynamic pressure, and heat flux with two parallel hierarchical series of field equations of balance type. The constitutive equations are determined explicitly by the thermal and caloric equations of state. The theory naturally includes the special case of rarefied polyatomic gases and, as a singular limit, the case of monatomic gases. This approach was applied also for derivation of 6 moments moments ET model which extends the Meixner's theory [16].

Our aim is to show that maximum entropy principle applied to kinetic model for polyatomic gases yields appropriate macroscopic balance laws for 14 independent fields, and presents a natural generalization of the same procedure applied for monatomic gases. Principal novelty, which is in accordance with the conjecture of ET of real gases [13], is that we obtain two hierarchies of transfer equations for moments, in contrast to one hierarchy in the case of monatomic gases. One of them is related to classical moments of distribution function, i.e. "momentum-like" moments. Another independent hierarchy, which starts with the second order moment corresponding to internal energy density, is expanded into transfer equations for "energy-like" moments. This hierarchy, however, cannot be merged into the first one since internal energy density is no longer twice the trace of the classical second order moment, as it is in monatomic case.

The paper is organized as follows. The Section 2 contains brief review 
of classical results related to application of the maximum entropy principle in kinetic theory of monatomic gases. In Section 3 the basic features of the model for polyatomic gases will be discussed at the Euler level. In particular, an equilibrium distribution function will be derived using maximum entropy principle, coinciding with the equilibrium distribution deduced in [1] by means of $H$-theorem, with accompanying Euler equations. This will also be the preparatory material for the next two sections where new nonequilibrium results will be given. Namely, Section 4 will contain exposition of the main idea of the paper - to construct two independent hierarchies of moment equations, "momentum" and "energy" one. The Section 5 will be focused on 14 moments approximation of velocity distribution function. First, the non-equilibrium velocity distribution function will be derived by means of maximum entropy principle. Second, corresponding set of balance laws (field equations) as transfer equations for moments will be derived in the spirit of procedure described in Section 4, which proves the complete consistence between the entropy principle of ET for real gases and MEP for polyatomic gases. This part of the study also contains novel results regarding the source terms and transport coefficients in balance laws for momentum flux (i.e. pressure tensor) and energy (heat) flux. Finally, entropy density and entropy flux will be determined for polyatomic gases both in equilibrium and non-equilibrium case. It is shown that Gibbs relation holds for equilibrium distribution function, whereas entropy density and entropy flux represent generalization of Grad's ones, since they comprise dynamic pressure. The section finishes with discussion on the qualitative analysis in the framework of symmetric hyperbolic systems. At the end, some remarks about further problems will be given.

\section{Moment equations and entropy maximization}

The kinetic theory of gases assumes that the state of the monatomic gas is described by the velocity distribution function $f(t, \mathbf{x}, \boldsymbol{\xi})$. Then $f(t, \mathbf{x}, \boldsymbol{\xi}) d \mathbf{x} d \boldsymbol{\xi}$ is the number of atoms in the volume $d \mathbf{x} d \boldsymbol{\xi}$ of phase space centered at $(\mathbf{x}, \boldsymbol{\xi}) \in \mathbb{R}^{3} \times \mathbb{R}^{3}$. The time rate of change of the velocity distribution function in the absence of external forces is determined by the Boltzmann equation

$$
\partial_{t} f+\xi_{j} \partial_{j} f=Q(f),
$$

where the collision integral $Q(f)$ determines the collision rate of change of distribution function. The symbols $\partial_{t}$ and $\partial_{j}$ denote partial derivatives with 
respect to time $t$ and space variables $x_{j}$, respectively ${ }^{1}$. Finding solution of (1) is extremely complicated and laborious task. Moreover, one is usually interested in evolution of macroscopic observable quantities under special conditions and exact solution is not even necessary. One way to circumvent direct solution of the Boltzmann equation is to define certain number of macroscopic quantities through averaging $f$ over the velocity space and to determine appropriate governing equations for them. In fact, these macroscopic quantities are the moments of distribution function:

$$
F_{i_{1} \ldots i_{n}}=\int_{\mathbb{R}^{3}} m \xi_{i_{1}} \cdots \xi_{i_{n}} f d \boldsymbol{\xi}, \quad\left(i_{k} \in\{1,2,3\}, \forall k \in \mathbb{N}\right) .
$$

Transfer equations for moments have the form of balance laws:

$$
\partial_{t} \mathbf{F}+\partial_{j} \mathbf{F}_{j}=\mathbf{P}
$$

where, the densities $\mathbf{F}$, the fluxes $\mathbf{F}_{j}$ and the productions $\mathbf{P}$ are:

$$
\begin{aligned}
\mathbf{F}(t, \mathbf{x}) & =\int_{\mathbb{R}^{3}} \boldsymbol{\Psi}(\boldsymbol{\xi}) f d \boldsymbol{\xi}, \\
\mathbf{F}_{j}(t, \mathbf{x}) & =\int_{\mathbb{R}^{3}} \xi_{j} \boldsymbol{\Psi}(\boldsymbol{\xi}) f d \boldsymbol{\xi}, \\
\mathbf{P}(t, \mathbf{x}) & =\int_{\mathbb{R}^{3}} \boldsymbol{\Psi}(\boldsymbol{\xi}) Q(f) d \boldsymbol{\xi},
\end{aligned}
$$

with

$$
\boldsymbol{\Psi}(\boldsymbol{\xi})=m\left(\begin{array}{c}
1 \\
\xi_{i_{1}} \\
\xi_{i_{1}} \xi_{i_{2}} \\
\vdots \\
\xi_{i_{1}} \cdots \xi_{i_{n}} \\
\vdots
\end{array}\right)
$$

The Boltzmann equation (1) is in this way substituted by an infinite sequence of balance laws for the moments (2). Notable property of this sequence is that the flux in moment equation of tensorial order $n$ becomes the density in the moment equation of order $n+1$. The first five balance laws, obtained

\footnotetext{
${ }^{1}$ Throughout the paper summation with respect to repeated indices will be assumed.
} 
for collision invariants $\left(m, m \xi_{i},(1 / 2) m|\boldsymbol{\xi}|^{2}\right)$, have the physical meaning of conservation laws of mass, momentum and energy, since the first 5 production terms are annihilated.

The hierarchy of macroscopic equations (2) is usually truncated at some order of moments, say $N$, and the closure of the system and the accuracy of the solution of the truncated system strongly depends upon proper choice of the distribution function. One method which provides appropriate solution of this problem is the maximization of entropy. It is well known that every solution $f$ of the Boltzmann equation (1) satisfies the supplementary entropy balance law:

$$
\partial_{t} h+\partial_{j} h_{j}=\Sigma \geq 0
$$

where

$h=-k \int_{\mathbb{R}^{3}} f \log f d \boldsymbol{\xi}, \quad h_{j}=-k \int_{\mathbb{R}^{3}} \xi_{j} f \log f d \boldsymbol{\xi}, \quad \Sigma=-k \int_{\mathbb{R}^{3}} Q(f) \log f d \boldsymbol{\xi}$,

$k$ being the Boltzmann constant. This result, in a bit different form (with factor $-k$ dropped), is known as $H$-theorem, equality being satisfied when $f$ is the Maxwellian distribution. On the other hand, it was shown that the local Maxwellian distribution (with hydrodynamic variables which depend on $t$ and $\mathbf{x}$ ), as well as the Grad's distribution, maximizes the physical entropy $h$.

These results motivated formulation of the maximum entropy principle. It states that actual distribution function $f$ is the one which maximizes the physical entropy:

$$
h=-k \int_{\mathbb{R}^{3}} f \log f d \boldsymbol{\xi} \rightarrow \max ,
$$

under the constraints that its moments are prescribed:

$$
\mathbf{F}^{(N)}(t, \mathbf{x})=\int_{\mathbb{R}^{3}} \Psi^{(N)}(\boldsymbol{\xi}) f(t, \mathbf{x}, \boldsymbol{\xi}) d \boldsymbol{\xi}
$$

where $\mathbf{F}^{(N)}$ is the truncated vector of moments up to tensorial order $N$, and $\boldsymbol{\Psi}^{(N)}$ is the vector $\boldsymbol{\Psi}$ truncated at order $N$. Thus, the approximate distribution function comes out as solution of a variational problem with constraints.

The problem can put on physical ground in the following way: entropy is related to probability of reaching certain state, and maximization of entropy is the search for the most probable velocity distribution function. On the 
other hand, the only information we get about the system is its macroscopic state, described by the moments (6) of the distribution function. Therefore, we seek a distribution function $f$ within the set of functions compatible with a given macroscopic state (set of admissible functions) which maximizes the entropy ${ }^{2}$.

The variational problem (5) with the constraints (6) is solved by the method of Lagrange multipliers and yields the following solution

$$
f(t, \mathbf{x}, \boldsymbol{\xi})=\exp (-1-\chi / k), \quad \chi=\boldsymbol{\lambda}^{(N)}(t, \mathbf{x}) \cdot \Psi^{(N)}(\boldsymbol{\xi}) .
$$

It is important to note that the Lagrange multipliers, and consequently the distribution function, depend on $t$ and $\mathbf{x}$ through the moments, $\boldsymbol{\lambda}^{(N)} \equiv$ $\boldsymbol{\lambda}^{(N)}\left(\mathbf{F}^{(N)}(t, \mathbf{x})\right)$. For both mathematical (convergence) and physical reasons (processes not far from equilibrium), carefully explained in [17] §3.16, the maximizer (7) is expanded in the neighborhood of local equilibrium. Therefore, the distribution function can be approximated as:

$$
f=f_{M}\left(1-\frac{1}{k} \tilde{\boldsymbol{\lambda}}^{(N)} \cdot \Psi^{(N)}\right), \quad \tilde{\boldsymbol{\lambda}}^{(N)}=\boldsymbol{\lambda}^{(N)}-\boldsymbol{\lambda}_{E}^{(N)}
$$

where $f_{M}$ denotes the Maxwellian distribution in local equilibrium (the local Maxwellian) and $\boldsymbol{\lambda}_{E}^{(N)}$ are the Lagrange multipliers evaluated at local equilibrium state. It was proved in [18] that all the equilibrium multipliers vanish, except the first 5 which correspond to the first 5 moments, i.e. the hydrodynamic variables. Plugging (8) into (6) we obtain a linear algebraic system that permits to evaluate the Lagrange multipliers $\tilde{\boldsymbol{\lambda}}^{(N)}$ in terms of the densities $\mathbf{F}^{(N)}$ :

$$
\begin{aligned}
\mathbf{F}^{(N)}(t, \mathbf{x}) & -\mathbf{F}_{E}^{(N)}(t, \mathbf{x})= \\
& -\frac{1}{k} \int_{\mathbb{R}^{3}}\left(\tilde{\boldsymbol{\lambda}}^{(N)}(t, \mathbf{x}) \cdot \Psi^{(N)}(\boldsymbol{\xi})\right) \Psi^{(N)}(\boldsymbol{\xi}) f_{E}(t, \mathbf{x}, \boldsymbol{\xi}) d \boldsymbol{\xi}
\end{aligned}
$$

$\left(\mathbf{F}_{E}^{(N)}\right.$ denotes the equilibrium values). Inserting $\tilde{\boldsymbol{\lambda}}^{(N)}$ solution of (9) into (8), we obtain the explicit dependence of the truncated fluxes and source terms

$$
\mathbf{F}_{j}^{(N)}(t, \mathbf{x})=\int_{\mathbb{R}^{3}} \xi_{j} \boldsymbol{\Psi}^{(N)}(\boldsymbol{\xi}) f d \boldsymbol{\xi}, \quad \mathbf{P}^{(N)}(t, \mathbf{x})=\int_{\mathbb{R}^{3}} \xi_{j} \boldsymbol{\Psi}^{(N)}(\boldsymbol{\xi}) Q(f) d \boldsymbol{\xi},
$$

\footnotetext{
${ }^{2}$ To be more precise we have to write $f^{(N)}$ instead of $f$ because we have different $f$ changing $N$ but to avoid heavy notation we omit the index $N$. Nevertheless, it is important to note that $f^{(N)}$ is not solution of the Boltzmann equation (1) and the conjecture is that $f^{(N)}$ converge to $f$ when $N \rightarrow \infty$.
} 
on the densities and, as a consequence, the truncated system of moment equations:

$$
\partial_{t} \mathbf{F}^{(N)}+\partial_{j} \mathbf{F}_{j}^{(N)}=\mathbf{P}^{(N)},
$$

is a closed system in which the only unknowns are the densities.

It was shown by Boillat and Ruggeri [10] that the maximum entropy solution (8) of order $N$ generates the equivalent set of balance laws as extended thermodynamics of the same order in which it is required that the truncated system (10) satisfy the entropy principle (4) with entropy, entropy density and entropy production which depend on the truncated densities. It is also proved that the closed balance laws system can be put into symmetric hyperbolic form using the main field $\mathbf{u}^{\prime}$ which coincides with Lagrange multipliers $\boldsymbol{\lambda}^{(N)}$. Moreover, the same authors put in evidence that the moments closure gives a nesting theories of principal subsystems. In fact, it was proved in [18] that if the closure is obtained with two different truncation orders $M<N$, then the system of order $M$ is a principal subsystem of the system of order $N$. The main consequence is that maximum characteristic velocity does not decrease with the index $N$, and tends to infinity when $N \rightarrow \infty$ [10]. Generalization of this procedure for higher order expansions of distribution function was given in [19]. Recently, the maximum entropy principle was discussed in a broader context of evolution equations with source terms [20].

\section{Equilibrium distribution function for polyatomic gases}

Our first aim is to recover the equilibrium distribution function and appropriate field equations for hydrodynamic variables (i.e. transfer equations for moments) via MEP. We shall, therefore, briefly describe the kinetic model for polyatomic gases and point out the important consequences related to internal energy density.

The basic feature of the kinetic model for polyatomic gases proposed in [1], and later on applied to chemically reacting mixtures [21], is the presence of a single continuous internal energy parameter $I \geq 0$. It represents communicable internal energy of the molecules in collision, i.e. it captures the influence of internal degrees of freedom on energy exchange during collisions. To that end, the conservation laws of momentum and energy during collisions 
read:

$$
\begin{aligned}
\boldsymbol{\xi}^{\prime}+\boldsymbol{\xi}_{*}^{\prime} & =\boldsymbol{\xi}+\boldsymbol{\xi}_{*}, \\
\frac{1}{2} m\left|\boldsymbol{\xi}^{\prime}\right|^{2}+\frac{1}{2} m\left|\boldsymbol{\xi}_{*}^{\prime}\right|^{2}+I^{\prime}+I_{*}^{\prime} & =\frac{1}{2} m|\boldsymbol{\xi}|^{2}+\frac{1}{2} m\left|\boldsymbol{\xi}_{*}\right|^{2}+I+I_{*},
\end{aligned}
$$

where $|\boldsymbol{\xi}|=\left(\xi_{i} \xi_{i}\right)^{1 / 2}$. The post-collisional quantities are described by the socalled Borgnakke-Larsen procedure, based on a repartition of the kinetic and internal energy [12], [1], [21]. We pass to the reference frame of the center of mass and write total energy of the incoming molecules, which is, by virtue of (11), the same as the one of the outgoing molecules:

$$
\varepsilon=\frac{1}{4} m\left|\boldsymbol{\xi}-\boldsymbol{\xi}_{*}\right|^{2}+I+I_{*}=\frac{1}{4} m\left|\boldsymbol{\xi}^{\prime}-\boldsymbol{\xi}_{*}^{\prime}\right|^{2}+I^{\prime}+I_{*}^{\prime} .
$$

Then, with a help of parameter $R \in[0,1]$, we attribute a part of total energy of the outgoing molecules to their kinetic energy, and the rest to their internal energy:

$$
\begin{aligned}
R \varepsilon & =\frac{1}{4} m\left|\boldsymbol{\xi}^{\prime}-\boldsymbol{\xi}_{*}^{\prime}\right|^{2} \\
(1-R) \varepsilon & =I^{\prime}+I_{*}^{\prime} .
\end{aligned}
$$

To distribute the internal energy itself between the two outgoing molecules, we introduce a new parameter $r \in[0,1]$ and write

$$
I^{\prime}=r(1-R) \varepsilon, \quad I_{*}^{\prime}=(1-r)(1-R) \varepsilon .
$$

Finally, as collisions between molecules are of specular reflection type, we parameterize (12) by a unitary vector $\boldsymbol{\omega} \in S^{2}$ :

$$
\boldsymbol{\xi}^{\prime}-\boldsymbol{\xi}_{*}^{\prime}=\sqrt{\frac{4 R \varepsilon}{m}} T_{\boldsymbol{\omega}}\left[\frac{\boldsymbol{\xi}-\boldsymbol{\xi}_{*}}{\left|\boldsymbol{\xi}-\boldsymbol{\xi}_{*}\right|}\right]
$$

where $T_{\boldsymbol{\omega}}$ is the symmetry with respect to the plane $\{\boldsymbol{\omega}\}^{\perp}$, i.e.

$$
T_{\boldsymbol{\omega}} \boldsymbol{z}=\boldsymbol{z}-2(\boldsymbol{\omega} \cdot \boldsymbol{z}) \boldsymbol{\omega}, \quad \forall \boldsymbol{z} \in \mathbb{R}^{3} .
$$

Coming back into the laboratory reference frame, we end up with expressions for post-collisional velocities:

$$
\boldsymbol{\xi}^{\prime}=\frac{\boldsymbol{\xi}+\boldsymbol{\xi}_{*}}{2}+\sqrt{\frac{R \varepsilon}{m}} T_{\boldsymbol{\omega}}\left[\frac{\boldsymbol{\xi}-\boldsymbol{\xi}_{*}}{\left|\boldsymbol{\xi}-\boldsymbol{\xi}_{*}\right|}\right], \quad \boldsymbol{\xi}_{*}^{\prime}=\frac{\boldsymbol{\xi}+\boldsymbol{\xi}_{*}}{2}-\sqrt{\frac{R \varepsilon}{m}} T_{\omega}\left[\frac{\boldsymbol{\xi}-\boldsymbol{\xi}_{*}}{\left|\boldsymbol{\xi}-\boldsymbol{\xi}_{*}\right|}\right] .
$$


As a consequence of considering one additional parameter, velocity distribution function $f(t, \mathbf{x}, \boldsymbol{\xi}, I)$ is defined on extended domain $[0, \infty) \times \mathbb{R}^{3} \times$ $\mathbb{R}^{3} \times[0, \infty)$. Its rate of change is determined by the Boltzmann equation which has the same form as (1) but collision integral $Q(f)$ takes into account the influence of internal degrees of freedom through collisional cross section. Collision invariants for this model form a 5 -vector:

$$
\boldsymbol{\psi}(\boldsymbol{\xi}, I)=m\left(1, \xi_{i},|\boldsymbol{\xi}|^{2}+2 \frac{I}{m}\right)^{T},
$$

which lead to hydrodynamic variables in the form:

$$
\left(\begin{array}{c}
\rho \\
\rho u_{i} \\
\rho|\mathbf{u}|^{2}+2 \rho \varepsilon
\end{array}\right)=\int_{\mathbb{R}^{3}} \int_{0}^{\infty} \boldsymbol{\psi}(\boldsymbol{\xi}, I) f(t, \mathbf{x}, \boldsymbol{\xi}, I) \varphi(I) d I d \boldsymbol{\xi},
$$

where $\rho, \mathbf{u}$ and $\varepsilon$ are mass density, hydrodynamic velocity and internal energy, respectively. A non-negative measure $\varphi(I) d I$ is property of the model aimed at recovering classical caloric equation of state for polyatomic gases in equilibrium. Entropy is defined by the following relation:

$$
h=-k \int_{\mathbb{R}^{3}} \int_{0}^{\infty} f \log f \varphi(I) d I d \boldsymbol{\xi} .
$$

We shall introduce the peculiar velocity:

$$
\mathrm{C}=\boldsymbol{\xi}-\mathbf{u}
$$

and rewrite the Eq. (14) in terms of it. Then:

$$
\left(\begin{array}{c}
\rho \\
0_{i} \\
2 \rho \varepsilon
\end{array}\right)=\int_{\mathbb{R}^{3}} \int_{0}^{\infty} m\left(\begin{array}{c}
1 \\
C_{i} \\
|\mathbf{C}|^{2}+2 I / m
\end{array}\right) f(t, \mathbf{x}, \mathbf{C}, I) \varphi(I) d I d \mathbf{C} .
$$

Note that the internal energy density can be divided into the translational part $\rho \varepsilon_{T}$ and part related to the internal degrees of freedom $\rho \varepsilon_{I}$ :

$$
\begin{aligned}
\rho \varepsilon_{T} & =\int_{\mathbb{R}^{3}} \int_{0}^{\infty} \frac{1}{2} m|\mathbf{C}|^{2} f(t, \mathbf{x}, \mathbf{C}, I) \varphi(I) d I d \mathbf{C}, \\
\rho \varepsilon_{I} & =\int_{\mathbb{R}^{3}} \int_{0}^{\infty} I f(t, \mathbf{x}, \mathbf{C}, I) \varphi(I) d I d \mathbf{C} .
\end{aligned}
$$


The former can be related to kinetic temperature in the following way:

$$
\varepsilon_{T}=\frac{3}{2} \frac{k}{m} T
$$

whereas the latter should determine the contribution of internal degrees of freedom to internal energy of a polyatomic gas.

The maximum entropy principle is expressed in terms of the following variational problem: determine the velocity distribution function $f(t, \mathbf{x}, \mathbf{C}, I)$ such that $h \rightarrow \max$, subject to the constraints (14), or equivalently, due to Galilean invariance, to the constraints (17). The solution is given as follows.

Statement 1. The distribution function which maximizes the entropy (15) subject to the constraints (17) has the form:

$$
f_{E}=\frac{\rho}{m q(T)}\left(\frac{m}{2 \pi k T}\right)^{3 / 2} \exp \left\{-\frac{1}{k T}\left(\frac{1}{2} m|\mathbf{C}|^{2}+I\right)\right\}
$$

where

$$
q(T)=\int_{0}^{\infty} \exp \left(-\frac{I}{k T}\right) \varphi(I) d I .
$$

The proof of the statement relies on application of Lagrange multiplier method. To that end we shall introduce the vector of multipliers $\left(\lambda^{(0)}, \lambda_{i}^{(1)}, \mu^{(2)}\right)$. Superscript indicates the tensorial order of the moment to which the multiplier is related. The appropriate extended functional is defined as follows:

$\mathcal{L}=\int_{\mathbb{R}^{3}} \int_{0}^{\infty}\left\{-k f \log f-m\left[\lambda^{(0)}+\lambda_{i}^{(1)} \xi_{i}+\mu^{(2)}\left(|\boldsymbol{\xi}|^{2}+2 \frac{I}{m}\right)\right] f\right\} \varphi(I) d I d \boldsymbol{\xi}$.

Since $\mathcal{L}$ is a scalar, it must retain the same value also for zero hydrodynamic velocity $\mathbf{u}=\mathbf{0}$, due to the Galilean invariance. Therefore:

$\mathcal{L}=\int_{\mathbb{R}^{3}} \int_{0}^{\infty}\left\{-k f \log f-m\left[\hat{\lambda}^{(0)}+\hat{\lambda}_{i}^{(1)} C_{i}+\hat{\mu}^{(2)}\left(|\mathbf{C}|^{2}+2 \frac{I}{m}\right)\right] f\right\} \varphi(I) d I d \mathbf{C}$,

where the hat denotes the Lagrange multipliers evaluated for $\mathbf{u}=\mathbf{0}$. Comparison between (22) and (23) yields the relations:

$$
\lambda^{(0)}=\hat{\lambda}^{(0)}-\hat{\lambda}_{i}^{(1)} u_{i}+\hat{\mu}^{(2)}|\mathbf{u}|^{2} ; \quad \lambda_{i}^{(1)}=\hat{\lambda}_{i}^{(1)}-2 \hat{\mu}^{(2)} u_{i} ; \quad \mu^{(2)}=\hat{\mu}^{(2)}
$$


which dictate the velocity dependence of Lagrange multipliers in accordance with the general results of Galilean invariance [22].

The Euler-Lagrange equation $\delta \mathcal{L} / \delta f=0$ leads to the following form of solution:

$$
f=\exp \left\{-1-\frac{m}{k}\left[\hat{\lambda}^{(0)}+\hat{\lambda}_{i}^{(1)} C_{i}+\hat{\mu}^{(2)}\left(|\mathbf{C}|^{2}+2 \frac{I}{m}\right)\right]\right\} .
$$

Plugging the solution into the constraints (17), with the help of (19), one determines the zero velocity Lagrange multipliers in terms of the hydrodynamic variables:

$$
\exp \left(-1-\frac{m}{k} \hat{\lambda}^{(0)}\right)=\frac{\rho}{m q(T)}\left(\frac{m}{2 \pi k T}\right)^{3 / 2}, \quad \hat{\lambda}_{i}^{(1)}=0, \quad \hat{\mu}^{(2)}=\frac{1}{2 T},
$$

with $q(T)$ is defined by (21), which completes the proof. Notice that using (24) and (25) we can have the explicit form of the Lagrange multipliers (i.e. the main field) that symmetrize the system and coincide with the Godunov variables [23]:

$$
\lambda^{(0)}=\frac{1}{T}\left(-G+\frac{1}{2}|\mathbf{u}|^{2}\right) ; \quad \lambda_{i}^{(1)}=-\frac{u_{i}}{T} ; \quad \mu^{(2)}=\frac{1}{2 T},
$$

where $G$ denotes the chemical potential.

The velocity distribution (20) is generalization of the classical Maxwellian equilibrium distribution in the case of polyatomic gases. It was derived in [1] and [21] by means of the $H$-theorem. However, the model chosen to describe the polyatomic structure, i.e. measure $\varphi(I) d I$, determines (20) through $q(T)$.

The structure of weighting function $\varphi(I)$ is determined such that it is possible to recover caloric equation of state for polyatomic gases. If $D$ is the number of degrees of freedom of a molecule, it can be shown that $\varphi(I)=I^{\alpha}$ leads to appropriate caloric equation in equilibrium provided:

$$
\alpha=\frac{D-5}{2} \text {. }
$$

Statement 2. In equilibrium, the internal energy $\varepsilon_{I}$ has the following form:

$$
\left.\varepsilon_{I}\right|_{E}=\frac{\rho}{m} \frac{\eta_{1}(T)}{q(T)}, \quad \eta_{1}(T)=\int_{0}^{\infty} I \varphi(I) \exp \left(-\frac{I}{k T}\right) d I
$$


Moreover, if weighting function is chosen to be $\varphi(I)=I^{\alpha}$, internal energy of a polyatomic gas in equilibrium reads:

$$
\left.\varepsilon\right|_{E}=\left(\frac{5}{2}+\alpha\right) \frac{k}{m} T, \quad \alpha>-1
$$

To prove (27) one just has to put the equilibrium distribution (20) into $(18)_{2}$. Furthermore, choosing $\varphi(I)=I^{\alpha}$, and taking into account the identity:

$$
\eta_{\beta}(T)=\int_{0}^{\infty} I^{\beta} \varphi(I) \exp \left(-\frac{I}{k T}\right) d I=(k T)^{\beta+1+\alpha} \Gamma(\beta+1+\alpha),
$$

for $\beta \geq 0$, auxiliary functions can be expressed in terms of Gamma function:

$$
q(T)=\eta_{0}(T)=(k T)^{1+\alpha} \Gamma(1+\alpha), \quad \eta_{1}(T)=(k T)^{2+\alpha} \Gamma(2+\alpha),
$$

with overall restriction $\alpha>-1$. With the help of relation $\Gamma(z+1)=z \Gamma(z)$ one obtains $\eta_{1}(T) / q(T)=(1+\alpha) k T$, which in turn leads to (28) by combining with translational energy (19).

The relation between $\alpha$ and $D$ (26) follows directly from comparison between (28) and well-know caloric equation for polyatomic gases:

$$
\left.\varepsilon\right|_{E}=\frac{D}{2} \frac{k}{m} T
$$

Observe that model for a monatomic gas $(D=3)$ cannot be recovered from the one with continuous internal energy, since the value of parameter $\alpha$ in monatomic case violates the overall restriction $\alpha>-1$.

If hydrodynamic variables $\rho, \mathbf{u}$ and $T$ in (20) are constants, distribution function identically satisfies the Boltzmann equation. If they are functions of $(t, \mathbf{x}) \in(0, \infty] \times \mathbb{R}^{3}$, we have so-called local Maxwellian distribution, but the hydrodynamic variables - moments of distribution function - cannot be arbitrary: they have to satisfy the transfer equations for moments. These equations have the form (10) for $\boldsymbol{\Psi}$ being substituted by collision invariants (13). As a matter of fact, they are the Euler gas dynamics equations for polyatomic gases.

Statement 3. If (20) is local equilibrium distribution with $\rho \equiv \rho(t, \mathbf{x}), \mathbf{u} \equiv$ $\mathbf{u}(t, \mathbf{x})$ and $T \equiv T(t, \mathbf{x})$, then the hydrodynamic variables $\rho$, $\mathbf{u}$ and $T$ satisfy 
the following systems of equations:

$$
\begin{aligned}
& \partial_{t} \rho+\partial_{j}\left(\rho u_{j}\right)=0, \\
& \partial_{t}\left(\rho u_{i}\right)+\partial_{j}\left(\rho u_{i} u_{j}+p \delta_{i j}\right)=0 \\
& \partial_{t}\left(\frac{1}{2} \rho|\mathbf{u}|^{2}+\rho \varepsilon\right)+\partial_{j}\left\{\left(\frac{1}{2} \rho|\mathbf{u}|^{2}+\rho \varepsilon+p u_{j}\right)\right\}=0
\end{aligned}
$$

where $\delta_{i j}$ is Kronecker delta, $p=(k / m) \rho T$ and $\varepsilon$ is determined by (28).

Equations (30) are obtained as moment equations of the distribution function in the form (10) with:

$$
\begin{aligned}
\mathbf{F}^{(2)} & =\int_{\mathbb{R}^{3}} \int_{0}^{\infty} \boldsymbol{\psi}(\boldsymbol{\xi}, I) f_{E} I^{\alpha} d I d \boldsymbol{\xi} \\
\mathbf{F}_{j}^{(2)} & =\int_{\mathbb{R}^{3}} \int_{0}^{\infty} \xi_{j} \boldsymbol{\psi}(\boldsymbol{\xi}, I) f_{E} I^{\alpha} d I d \boldsymbol{\xi} \\
\mathbf{P}^{(2)} & =\int_{\mathbb{R}^{3}} \int_{0}^{\infty} \boldsymbol{\psi}(\boldsymbol{\xi}, I) Q\left(f_{E}\right) I^{\alpha} d I d \boldsymbol{\xi}=\mathbf{0},
\end{aligned}
$$

for $\boldsymbol{\psi}(\boldsymbol{\xi}, I)$ defined by (13) and $N=2$. In fact, moments and their fluxes read:

$$
\mathbf{F}^{(2)}=\left(\begin{array}{c}
\rho \\
\rho u_{i} \\
\rho|\mathbf{u}|^{2}+2 \rho \varepsilon
\end{array}\right), \quad \mathbf{F}_{j}^{(2)}=\left(\begin{array}{c}
\rho u_{j} \\
\rho u_{i} u_{j}+p_{i j} \\
\left(\rho|\mathbf{u}|^{2}+2 \rho \varepsilon\right) u_{j}+2 p_{i j} u_{i}+2 q_{j}
\end{array}\right)
$$

where $p_{i j}$ and $q_{i}$ are pressure tensor and heat flux, respectively. When they are evaluated as fluxes of local Maxwellian (20) one obtains:

$$
\begin{aligned}
p_{i j} & =\int_{\mathbb{R}^{3}} \int_{0}^{\infty} m C_{i} C_{j} f_{E} I^{\alpha} d I d \mathbf{C}=\frac{k}{m} \rho T \delta_{i j}=p \delta_{i j}, \\
q_{j} & =\int_{\mathbb{R}^{3}} \int_{0}^{\infty} C_{j}\left(\frac{1}{2} m|\mathbf{C}|^{2}+I\right) f_{E} I^{\alpha} d I d \mathbf{C}=0,
\end{aligned}
$$

which completes the proof. Note that equations (30) have the same form in the case of monatomic gases, except for the fact that internal energy in monatomic case is strictly $\varepsilon=\frac{3}{2} \frac{k}{m} T$. 


\section{The hierarchy of the moment equations for polyatomic gases}

The derivation of macroscopic equations at Euler lever is rather straightforward. One takes the moments of local Maxwellian against collision invariants and obtains the conservation laws of mass, momentum and energy. The closure of the system is achieved due to the fact that non-equilibrium fluxes - pressure tensor and heat flux - vanish in local equilibrium state, whereas pressure and internal energy are uniquely determined.

If non-equilibrium effects are to be taken into account in polyatomic gases, one faces the problem at the very first step. Although the model with continuous internal energy parameter helped to recover the caloric equation of state, nice hierarchical structure, present in Eqs. (2)-(3) for monatomic gases, is destroyed. It is a consequence of the structure of collision invariants (13). The energy is not equal to the half of the trace of the second order moment anymore due to the presence of additional parameter $I$. Thus, the hierarchy of moments equations cannot be constructed in the same fashion as in the monatomic case. Consequently, Grad's procedure cannot be generalized in a straightforward manner. This difficulty was overcome in [24]. Main idea, already anticipated in [1], was to extend the state space of the molecule in order to treat additional parameter as velocity in such a higher-dimensional space. This procedure yielded hierarchy which is much alike the one for monatomic gases and allowed direct application of Grad's method.

Our analysis will pursue in different direction. In classical continuum mechanics the conservation laws of momentum and energy are independent physical laws as long as changes of internal energy of the medium occur. Motivated by the idea stated in the paper [13], we shall generalize this feature to moment equations for polyatomic gases by constructing two independent hierarchies. One will be much alike classical "momentum" hierarchy of monatomic gases (F-hierarchy); the other one, "energy" hierarchy, commences with the moment related to energy collision invariant and proceeds with standard increase of the order through multiplication by velocities $(G-h i e r a r c h y)$. They read:

$$
\partial_{t} \mathbf{F}+\partial_{j} \mathbf{F}_{j}=\mathbf{P}, \quad \partial_{t} \mathbf{G}+\partial_{j} \mathbf{G}_{j}=\mathbf{Q} .
$$


The moments, fluxes and productions of $F$-hierarchy are defined as:

$$
\begin{aligned}
\mathbf{F}(t, \mathbf{x}) & =\int_{\mathbb{R}^{3}} \int_{0}^{\infty} \boldsymbol{\Psi}(\boldsymbol{\xi}) f \varphi(I) d I d \boldsymbol{\xi}, \\
\mathbf{F}_{j}(t, \mathbf{x}) & =\int_{\mathbb{R}^{3}} \int_{0}^{\infty} \xi_{j} \boldsymbol{\Psi}(\boldsymbol{\xi}) f \varphi(I) d I d \boldsymbol{\xi}, \\
\mathbf{P}(t, \mathbf{x}) & =\int_{\mathbb{R}^{3}} \int_{0}^{\infty} \boldsymbol{\Psi}(\boldsymbol{\xi}) Q(f) \varphi(I) d I d \boldsymbol{\xi},
\end{aligned}
$$

with:

$$
\boldsymbol{\Psi}(\boldsymbol{\xi})=m\left(\begin{array}{c}
1 \\
\xi_{i_{1}} \\
\xi_{i_{1}} \xi_{i_{2}} \\
\vdots \\
\xi_{i_{1}} \cdots \xi_{i_{n}} \\
\vdots
\end{array}\right)
$$

The moments, fluxes and productions of $G$-hierarchy are defined as:

$$
\begin{aligned}
\mathbf{G}(t, \mathbf{x}) & =\int_{\mathbb{R}^{3}} \int_{0}^{\infty} \boldsymbol{\Theta}(\boldsymbol{\xi}, I) f \varphi(I) d I d \boldsymbol{\xi}, \\
\mathbf{G}_{j}(t, \mathbf{x}) & =\int_{\mathbb{R}^{3}} \int_{0}^{\infty} \xi_{j} \boldsymbol{\Theta}(\boldsymbol{\xi}, I) f \varphi(I) d I d \boldsymbol{\xi}, \\
\mathbf{Q}(t, \mathbf{x}) & =\int_{\mathbb{R}^{3}} \int_{0}^{\infty} \boldsymbol{\Theta}(\boldsymbol{\xi}, I) Q(f) \varphi(I) d I d \boldsymbol{\xi},
\end{aligned}
$$

with:

$$
\boldsymbol{\Theta}(\boldsymbol{\xi}, I)=m\left(\begin{array}{c}
|\boldsymbol{\xi}|^{2}+2 \frac{I}{m} \\
\left(|\boldsymbol{\xi}|^{2}+2 \frac{I}{m}\right)^{2} \xi_{k_{1}} \\
\left(|\boldsymbol{\xi}|^{2}+2 \frac{I}{m}\right)^{2} \xi_{k_{1}} \xi_{k_{2}} \\
\vdots \\
\left(|\boldsymbol{\xi}|^{2}+2 \frac{I}{m}\right) \xi_{k_{1}} \cdots \xi_{k_{m}} \\
\vdots
\end{array}\right)
$$

i.e.

$$
\boldsymbol{\Theta}(\boldsymbol{\xi}, I)=\left(2 \frac{I}{m}+|\boldsymbol{\xi}|^{2}\right) \boldsymbol{\Psi}(\boldsymbol{\xi}) .
$$

Note that minimal order of the moment in $F$-hierarchy is 0 , while minimal order in $G$-hierarchy is 2. Furthermore, due to the collision invariants the 
first two orders of productions in $F$-hierarchy are zero, as well as second (the lowest) order production in $G$-hierarchy. Finally, the property that flux in moment equation of order $n$ becomes the density in moment equation of order $n+1$ holds, albeit separately for $F$ - and for $G$-hierarchy.

Like in monatomic gases, infinite sequences of moment equations may be truncated to form a finite set of balance laws:

$$
\partial_{t} \mathbf{F}^{(N)}+\partial_{j} \mathbf{F}_{j}^{(N)}=\mathbf{P}^{(N)}, \quad \partial_{t} \mathbf{G}^{(M)}+\partial_{j} \mathbf{G}_{j}^{(M)}=\mathbf{Q}^{(M)} .
$$

for $N \geq 0$ and $M \geq 2$. Note that local equilibrium case, equations (30) from previous section, is recovered for $N=1$ and $M=2$, with closure achieved through the equilibrium distribution (20). For higher order approximations the closure problem remains open. In the next section we shall study the most interesting physical case of non-equilibrium 14 moments.

\section{The 14 moments system for polyatomic gases}

When the system is dissipative, the pressure tensor $p_{i j}$, or equivalently the stress tensor $t_{i j}=-p_{i j}$, have also a non-equilibrium part due to viscosity:

$$
p_{i j}=p \delta_{i j}-S_{i j}
$$

In the real gas, $S_{i j}$ have an isotropic part and a deviatoric part:

$$
S_{i j}=-\Pi \delta_{i j}-p_{\langle i j\rangle},
$$

where $\Pi$ is the so-called dynamic pressure, which vanishes in monatomic case, and $p_{\langle i j\rangle}$ is traceless part of the pressure tensor. Therefore, we have:

$$
p_{i j}=(p+\Pi) \delta_{i j}+p_{\langle i j\rangle} .
$$

Moreover, the heat flux $q_{i}$ does not vanish in non-equilibrium. The existence of the dynamic pressure implies that non-equilibrium state of real gas is described by 14 field variables, instead of 13 fields of monatomic gas.

The recent study of ET of real gases [13] suggested the following form of field equations governing evolution of 14 field variables (moments):

$$
\begin{array}{ll}
\partial_{t} F+\partial_{j} F_{j}=0, & \\
\partial_{t} F_{i_{1}}+\partial_{j} F_{i_{1} j}=0, & \partial_{t} G_{p p}+\partial_{j} G_{p p j}=0, \\
\partial_{t} F_{i_{1} i_{2}}+\partial_{j} F_{i_{1} i_{2} j}=P_{i_{1} i_{2}}, & \partial_{t} G_{p p k_{1}}+\partial_{j} G_{p p k_{1} j}=Q_{p p k_{1}},
\end{array}
$$


where $F$ is the mass density, $F_{i_{1}}$ is the momentum density, $G_{p p}$ is twice the total energy density, $F_{i_{1} i_{2}}$ is the momentum flux, and $G_{p p k_{1}}$ is twice the total energy flux; $F_{i_{1} i_{2} j}$ and $G_{p p k_{1} j}$ are the fluxes of $F_{i_{1} i_{2}}$ and $G_{p p k_{1}}$, respectively, and $P_{i_{1} i_{2}}$ and $Q_{p p k_{1}}$ are the productions with respect to $F_{i_{1} i_{2}}$ and $G_{p p k_{1}}$ respectively. It is inevitable that equations (34) perfectly fit into hierarchy of moment equations for rarefied polyatomic gas, if one choose $N=2$ and $M=3$ :

$$
\partial_{t} \mathbf{F}^{(2)}+\partial_{j} \mathbf{F}_{j}^{(2)}=\mathbf{P}^{(2)}, \quad \partial_{t} \mathbf{G}^{(3)}+\partial_{j} \mathbf{G}_{j}^{(3)}=\mathbf{Q}^{(3)} .
$$

The vectors of densities are:

$$
\begin{aligned}
& \mathbf{F}^{(2)}(t, \mathbf{x})=\left(\begin{array}{c}
F \\
F_{i_{1}} \\
F_{i_{1} i_{2}}
\end{array}\right)=\int_{\mathbb{R}^{3}} \int_{0}^{\infty} m\left(\begin{array}{c}
1 \\
\xi_{i_{1}} \\
\xi_{i_{1}} \xi_{i_{2}}
\end{array}\right) f(t, \mathbf{x}, \boldsymbol{\xi}, I) \varphi(I) d I d \boldsymbol{\xi} \\
& \mathbf{G}^{(3)}(t, \mathbf{x})=\left(\begin{array}{c}
G_{p p} \\
G_{p p k_{1}}
\end{array}\right)=\int_{\mathbb{R}^{3}} \int_{0}^{\infty} m\left(\begin{array}{c}
|\boldsymbol{\xi}|^{2}+2 \frac{I}{m} \\
\left(|\boldsymbol{\xi}|^{2}+2 \frac{I}{m}\right)^{2} \xi_{k_{1}}
\end{array}\right) f(t, \mathbf{x}, \boldsymbol{\xi}, I) \varphi(I) d I d \boldsymbol{\xi}
\end{aligned}
$$

In the sequel it will be assumed $\varphi(I)=I^{\alpha}, \alpha>-1$.

Inserting (16) into (36) we have the velocity dependence that it is in agreement with the Galilean invariance [13, 22]:

$$
\begin{aligned}
& F=\rho, \quad F_{i_{1}}=\rho u_{i_{1}}, \quad F_{i_{1} i_{2}}=\rho u_{i_{1}} u_{i_{2}}+p_{i_{1} i_{2}} \\
& G_{p p}=2 \rho \varepsilon+\rho|\mathbf{u}|, \quad G_{p p k_{1}}=\left(2 \rho \varepsilon+\rho|\mathbf{u}|^{2}\right) u_{k_{1}}+2 p_{i k_{1}} u_{i}+2 q_{i}
\end{aligned}
$$

with

$$
\begin{aligned}
& \left(\begin{array}{c}
\rho \\
0_{i} \\
p_{i j}
\end{array}\right)=\int_{\mathbb{R}^{3}} \int_{0}^{\infty} \Psi^{(2)}(\mathbf{C}) f(t, \mathbf{x}, \mathbf{C}, I) \varphi(I) d I d \mathbf{C}, \\
& \left(\begin{array}{c}
\rho \varepsilon \\
q_{i}
\end{array}\right)=\frac{1}{2} \int_{\mathbb{R}^{3}} \int_{0}^{\infty} \Theta^{(3)}(\mathbf{C}, I) f(t, \mathbf{x}, \mathbf{C}, I) \varphi(I) d I d \mathbf{C},
\end{aligned}
$$

where the following notation is used:

$$
\Psi^{(2)}(\mathbf{C})=m\left(\begin{array}{c}
1 \\
C_{i} \\
C_{i} C_{j}
\end{array}\right), \quad \Theta^{(3)}(\mathbf{C}, I)=m\left(\begin{array}{c}
|\mathbf{C}|^{2}+2 \frac{I}{m} \\
\left(|\mathbf{C}|^{2}+2 \frac{I}{m}\right)^{2} C_{i}
\end{array}\right) .
$$


Note that (36) determine 14 constraints for 14 scalar moments of distribution function, in contrast to 13 moments in monatomic gases. The number of constrained moments is increased since $F_{i i} \neq G_{i i}$ in polyatomic gases.

\subsection{The non-equilibrium distribution function}

For the entropy defined by (15) the following variational problem can be formulated, expressing the maximum entropy principle: determine the velocity distribution function $f(t, \mathbf{x}, \mathbf{C}, I)$ such that $h \rightarrow \max$, subject to constraints (37). The solution of the problem is as follows.

Statement 4. The velocity distribution function which maximizes the entropy (15) subject to constraints (37) for the choice of weighting function $\varphi(I)=I^{\alpha}$, has the form:

$$
\begin{aligned}
& f=f_{E}\left\{1-\frac{\rho}{p^{2}} q_{i} C_{i}+\frac{\rho}{p^{2}}\left[p_{\langle i j\rangle}-\left(\frac{5}{2}+\alpha\right)(1+\alpha)^{-1} \Pi \delta_{i j}\right] C_{i} C_{j}\right. \\
& \left.-\frac{3}{2(1+\alpha)} \frac{\rho}{p^{2}} \Pi\left(\frac{1}{2}|\mathbf{C}|^{2}+\frac{I}{m}\right)+\left(\frac{7}{2}+\alpha\right)^{-1} \frac{\rho^{2}}{p^{3}} q_{i}\left(\frac{1}{2}|\mathbf{C}|^{2}+\frac{I}{m}\right) C_{i}\right\}
\end{aligned}
$$

where $f_{E}$ is the equilibrium distribution (20) and $q(T)$ is the auxiliary function (21).

To solve the problem we shall introduce the following vectors of Lagrange multipliers:

$$
\boldsymbol{\lambda}^{(2)} \equiv\left(\lambda^{(0)}, \lambda_{i}^{(1)}, \lambda_{i j}^{(2)}\right), \quad \boldsymbol{\mu}^{(3)} \equiv\left(\mu^{(2)}, \mu_{i}^{(3)}\right)
$$

where $\boldsymbol{\lambda}^{(2)}$ corresponds to constraints $(36)_{1}$ and $\boldsymbol{\mu}^{(3)}$ corresponds to constraints $(36)_{2}$. The superscript indicates the order of the moment to which the multiplier is applied.

The extended functional for the constrained variational problem reads:

$$
\mathcal{L}=\int_{\mathbb{R}^{3}} \int_{0}^{\infty}\left\{-k f \log f-\boldsymbol{\lambda}^{(2)} \cdot \boldsymbol{\Psi}^{(2)}(\boldsymbol{\xi})-\boldsymbol{\mu}^{(3)} \cdot \boldsymbol{\Theta}^{(3)}(\boldsymbol{\xi}, I)\right\} \varphi(I) d \boldsymbol{\xi} .
$$

For Galilean invariance the functional is the same also for zero hydrodynamic velocity $(\mathbf{u}=\mathbf{0})$ and therefore

$$
\mathcal{L}=\int_{\mathbb{R}^{3}} \int_{0}^{\infty}\left\{-k f \log f-\hat{\boldsymbol{\lambda}}^{(2)} \cdot \mathbf{\Psi}^{(2)}(\mathbf{C})-\hat{\boldsymbol{\mu}}^{(3)} \cdot \mathbf{\Theta}^{(3)}(\mathbf{C}, I)\right\} \varphi(I) d \mathbf{C},
$$


where the following vectors of zero velocity Lagrange multipliers are introduced:

$$
\hat{\boldsymbol{\lambda}}^{(2)} \equiv\left(\hat{\lambda}^{(0)}, \hat{\lambda}_{i}^{(1)}, \hat{\lambda}_{i j}^{(2)}\right)^{T}, \quad \hat{\boldsymbol{\mu}}^{(3)} \equiv\left(\hat{\mu}^{(2)}, \hat{\mu}_{i}^{(3)}\right)^{T},
$$

and $\Psi^{(2)}(\mathbf{C})$ and $\boldsymbol{\Theta}^{(3)}(\mathbf{C}, I)$ are defined by (38). In the formulation of the variational problem it is sufficient to use the macroscopic quantities determined by peculiar velocity $\mathbf{C}$, rather than the molecular one $\boldsymbol{\xi}$. We observe that $\hat{\boldsymbol{\lambda}}^{(2)}$ corresponds to constraints $(37)_{1}$ and $\hat{\boldsymbol{\mu}}^{(3)}$ corresponds to constraints $(37)_{2}$.

The equivalence between (40) and (41) implies

$$
\boldsymbol{\lambda}^{(2)} \cdot \Psi^{(2)}(\mathbf{C}+\mathbf{u})+\boldsymbol{\mu}^{(3)} \cdot \boldsymbol{\Theta}^{(3)}(\mathbf{C}+\mathbf{u}, I)=\hat{\boldsymbol{\lambda}}^{(2)} \cdot \Psi^{(2)}(\mathbf{C})+\hat{\boldsymbol{\mu}}^{(3)} \cdot \boldsymbol{\Theta}^{(3)}(\mathbf{C}, I)
$$

for all $\mathbf{C} \in \mathbb{R}^{3}$. This implies the velocity dependence of Lagrange multipliers in accordance with the Galilean invariance [22]:

$$
\begin{aligned}
& \lambda^{(0)}=\hat{\lambda}^{(0)}-\hat{\lambda}_{i}^{(1)} u_{i}+\hat{\lambda}_{i j}^{(2)} u_{i} u_{j}+\hat{\mu}^{(2)}|\mathbf{u}|^{2}-\hat{\mu}_{j}^{(3)} u_{j}|\mathbf{u}|^{2}, \\
& \lambda_{i}^{(1)}=\hat{\lambda}_{i}^{(1)}-2 \hat{\lambda}_{i j}^{(2)} u_{j}-2 \hat{\mu}^{(2)} u_{i}+2 \hat{\mu}_{j}^{(3)} u_{i} u_{j}+\hat{\mu}_{j}^{(3)}|\mathbf{u}|^{2}, \\
& \lambda_{i j}^{(2)}=\hat{\lambda}_{i j}^{(2)}-\left(\hat{\mu}_{j}^{(3)} u_{i}+\hat{\mu}_{i}^{(3)} u_{j}\right)+\frac{4}{9} \hat{\mu}_{k}^{(3)} u_{k} \delta_{i j}, \\
& \mu^{(2)}=\hat{\mu}^{(2)}-\hat{\mu}_{j}^{(3)} u_{j}, \\
& \mu_{j}^{(3)}=\hat{\mu}_{j}^{(3)} .
\end{aligned}
$$

From (41) the solution of the Euler-Lagrange equation $\delta \mathcal{L} / \delta f=0$ is given by:

$$
f=\exp (-1-\chi / k), \quad \chi=\hat{\boldsymbol{\lambda}}^{(2)} \cdot \Psi^{(2)}(\mathbf{C})+\hat{\boldsymbol{\mu}}^{(3)} \cdot \boldsymbol{\Theta}^{(3)}(\mathbf{C}, I) .
$$

The apparent similarity between this solution and solution (7) in monatomic case draws our attention to the convergence problem. To ensure the convergence of moments of distribution function, we shall recourse to the approximate form of the maximizer by its expansion in the neighborhood of local equilibrium:

$$
f=f_{E}\left(1-\frac{1}{k}\left(\tilde{\boldsymbol{\lambda}}^{(2)} \cdot \mathbf{\Psi}^{(2)}+\tilde{\boldsymbol{\mu}}^{(3)} \cdot \mathbf{\Theta}^{(3)}\right)\right)
$$


where $f_{E}$ is the equilibrium distribution (20) and $\tilde{\boldsymbol{\lambda}}^{(2)}=\hat{\boldsymbol{\lambda}}^{(2)}-\hat{\boldsymbol{\lambda}}_{E}^{(2)}, \tilde{\boldsymbol{\mu}}^{(3)}=$ $\hat{\boldsymbol{\mu}}^{(3)}-\hat{\boldsymbol{\mu}}_{E}^{(3)}$, where subscript $E$ indicates the values of the Lagrange multipliers at the local equilibrium state.

Inserting (43) into the constraints (37) one immediately obtains $\tilde{\lambda}^{(0)}=0$, and taking into account (28) the following system of algebraic equations emerges:

$$
\begin{aligned}
& \tilde{\lambda}_{i i}^{(2)}+(5+2 \alpha) \tilde{\mu}^{(2)}=0, \\
& \tilde{\lambda}_{i}^{(1)}+(7+2 \alpha) \frac{p}{\rho} \tilde{\mu}_{i}^{(3)}=0, \\
& 2 \frac{p^{2}}{\rho} \tilde{\lambda}_{i j}^{(2)}-\left(\frac{k}{m} p-\frac{2 p^{2}}{\rho} \tilde{\mu}^{(2)}\right) \delta_{i j}=-\frac{k}{m} p_{i j}, \\
& \left(\frac{7}{2}+\alpha\right) \tilde{\lambda}_{i i}^{(2)}+\left[\frac{15}{2}+2(1+\alpha)(5+\alpha)\right] \tilde{\mu}^{(2)}=0, \\
& \left(\frac{7}{2}+\alpha\right) \frac{p^{2}}{\rho} \tilde{\lambda}_{i}^{(1)}+\left[\frac{35}{2}+2(1+\alpha)(7+\alpha)\right] \frac{p^{3}}{\rho^{2}} \tilde{\mu}_{i}^{(3)}=-\frac{k}{m} q_{i} .
\end{aligned}
$$

The equations $(44)_{1-3}$ come out from the constraints $(37)_{1}$, while $(44)_{4-5}$ come out from $(37)_{2}$. Simple analysis shows that $(44)_{1}$ and $(44)_{4}$ are linearly dependent. Taking the trace of $(44)_{3}$ one obtains:

$$
-2 \frac{p^{2}}{\rho} \tilde{\lambda}_{i i}^{(2)}+3\left(\frac{k}{m} p-\frac{2 p^{2}}{\rho} \tilde{\mu}^{(2)}\right)=\frac{k}{m} p_{i i}
$$

which in conjunction with $(44)_{1}$ gives:

$$
\frac{k}{m}\left(\frac{1}{3} p_{i i}-p\right)=\frac{4}{3} \frac{p^{2}}{\rho}(1+\alpha) \tilde{\mu}^{(2)}=\frac{k}{m} \Pi .
$$

By combining this result with $(44)_{3}$ and solving Eqs. $(44)_{2}$ and $(44)_{5}$, the following solution is obtained:

$$
\begin{aligned}
\tilde{\lambda}_{i}^{(1)} & =\frac{k}{m} \frac{\rho}{p^{2}} q_{i} \\
\tilde{\lambda}_{i j}^{(2)} & =-\frac{k}{m} \frac{\rho}{2 p^{2}}\left\{p_{\langle i j\rangle}+\left(\frac{5}{2}+\alpha\right)(1+\alpha)^{-1} \Pi \delta_{i j}\right\}, \\
\tilde{\mu}^{(2)} & =\frac{k}{m} \frac{3}{4(1+\alpha)} \frac{\rho}{p^{2}} \Pi, \\
\tilde{\mu}_{i}^{(3)} & =-\frac{k}{2 m}\left(\frac{7}{2}+\alpha\right)^{-1} \frac{\rho^{2}}{p^{3}} q_{i},
\end{aligned}
$$


and thus non-equilibrium velocity distribution (39) is obtained.

Remarkable property of real gases, anticipated at the beginning of the Section, is the existence of the dynamic pressure, which does not appear in monatomic gases. It manifests itself through Eq. (45), although its existence is anticipated in $(44)_{1}$. Namely, in the monatomic case, i.e. classical Grad's distribution, constraint $(37)_{1}$ yields $\tilde{\lambda}_{i i}^{(2)}=0$, and consequently $\Pi=0$. Additional term in $(44)_{1}$ is due to the presence of internal energy parameter $I$ in equilibrium distribution (20).

The important feature of the non-equilibrium distribution (39) is its compatibility with other distributions obtained in the context of polyatomic gases. Firstly, it reduces to velocity distribution obtained by Mallinger [24] for diatomic molecules $(\alpha=0)$. Further, a comparison could be made with semi-classical model of Wang Chang, Uhlenbeck and de Boer [25], which assumes that molecules can occupy discrete states of internal energy. It may be observed (see [11], Section 5.2) that the auxiliary function $q(T)$ takes the role of the partition function $\mathcal{Z}=\sum_{\alpha} \exp \left(-e_{\alpha} / k T\right)$. The complete compatibility with corresponding non-equilibrium function can be observed when $I$ is substituted by $e_{\alpha}$ and macroscopic quantities are defined by summation over $\alpha$ instead of integration over $I$.

\subsection{Non-convective fluxes and production terms}

The non-equilibrium distribution function (39) can be regarded as approximation of the exact solution of the Boltzmann equation expressed in terms of moments. Nevertheless, we have yet another benefit as well: it is a way by which closure of the transfer equations for moments is achieved. As already noticed, fluxes in transfer equations of order $n$ become densities in equations of order $n+1$. This leaves the fluxes in equations of order $n+1$ undetermined, as well as all the production terms. Maximization of entropy, subject to constraints (37), permits to calculate the fluxes:

$$
\mathbf{F}_{j}^{(2)}=\left(\begin{array}{c}
F_{i} \\
F_{i j} \\
F_{i j k}
\end{array}\right), \quad \mathbf{G}_{j}^{(3)}=\left(\begin{array}{c}
G_{p p i} \\
G_{p p i j}
\end{array}\right),
$$

where taking into account (16) and Galilean invariance:

$$
\begin{aligned}
F_{i j k} & =\rho u_{i} u_{j} u_{k}+u_{i} p_{j k}+u_{j} p_{k i}+u_{k} p_{i j}+p_{i j k}, \\
G_{\text {ppij }} & =\left(\rho|\mathbf{u}|^{2}+2 \rho \varepsilon\right) u_{i} u_{j}+2 u_{i} u_{k} p_{j k}+2 u_{j} u_{k} p_{i k}+\rho|\mathbf{u}|^{2} p_{i j}+2 q_{i j} .
\end{aligned}
$$


The non-convective fluxes are defined as:

$$
\begin{aligned}
p_{i j k} & =\int_{\mathbb{R}^{3}} \int_{0}^{\infty} m C_{i} C_{j} C_{k} f(t, \mathbf{x}, \mathbf{C}, I) \varphi(I) d I d \mathbf{C}, \\
q_{i j} & =\int_{\mathbb{R}^{3}} \int_{0}^{\infty}\left(\frac{1}{2} m|\mathbf{C}|^{2}+I\right) C_{i} C_{j} f(t, \mathbf{x}, \mathbf{C}, I) \varphi(I) d I d \mathbf{C} .
\end{aligned}
$$

Statement 5. The non-convective fluxes $p_{i j k}$ and $q_{i j}$ in 14 moments approximation have the following form:

$$
\begin{aligned}
p_{i j k} & =\left(\frac{7}{2}+\alpha\right)^{-1}\left(q_{i} \delta_{j k}+q_{j} \delta_{k i}+q_{k} \delta_{i j}\right), \\
q_{i j} & =\left(\frac{9}{2}+\alpha\right) \frac{p}{\rho} p_{i j}-\frac{p^{2}}{\rho} \delta_{i j} .
\end{aligned}
$$

Expressions (46) and (47) for non-convective fluxes are derived in a straightforward way by plugging (39) in the above given definitions.

It must be emphasized that structure of non-convective fluxes (46) and (47) implies genuine coupling of two hierarchies - heat flux $q_{i}$ appears in $F$-hierarchy, whereas pressure tensor $p_{i j}$ appears in $G$-hierarchy. It also resembles the structure of non-convective fluxes in Grad's approximation for monatomic gases and can be regarded as its generalization. However, it is not only the parameter $\alpha$ which brings the flavor of polyatomic gases, but also the pressure tensor whose trace contains dynamic pressure (apart from the ideal gas one).

Closure of the moments system (35) needs calculation of production terms:

$$
\begin{aligned}
& \mathbf{P}^{(2)}(t, \mathbf{x})=\int_{\mathbb{R}^{3}} \int_{0}^{\infty} m\left(\begin{array}{c}
1 \\
\xi_{i} \\
\xi_{i} \xi_{j}
\end{array}\right) Q(f) \varphi(I) d I d \boldsymbol{\xi}=\left(\begin{array}{c}
0 \\
0_{i} \\
P_{i j}
\end{array}\right) \\
& \mathbf{Q}^{(3)}(t, \mathbf{x})=\int_{\mathbb{R}^{3}} \int_{0}^{\infty} m\left(\begin{array}{c}
|\boldsymbol{\xi}|^{2}+2 \frac{I}{m} \\
\left(|\boldsymbol{\xi}|^{2}+2 \frac{I}{m}\right)
\end{array}\right) Q(f) \varphi(I) d I d \boldsymbol{\xi}=\left(\begin{array}{c}
0 \\
Q_{i}
\end{array}\right) .
\end{aligned}
$$

Zero entries appear due to collision invariants. Typical obstacle in calculation of production terms is complicated structure of collision integral $Q(f)$. In this study we shall assume the structure of collision integral given in [1] and 
[21]:

$$
\begin{aligned}
Q(f)(\boldsymbol{\xi}, I) & =\int_{\mathbb{R}^{3} \times \mathbb{R}_{+} \times[0,1]^{2} \times S^{2}}\left[f\left(\boldsymbol{\xi}^{\prime}, I^{\prime}\right) f\left(\boldsymbol{\xi}_{*}^{\prime}, I_{*}^{\prime}\right)-f(\boldsymbol{\xi}, I) f\left(\boldsymbol{\xi}_{*}, I_{*}\right)\right] \times \\
& \times \mathcal{B}\left(\xi, \xi_{*}, I, I_{*}, r, R, \boldsymbol{\omega}\right)(1-R)\left|\xi-\xi_{*}\right| \frac{1}{\varphi(I)} d \boldsymbol{\omega} d r d R d I_{*} d \boldsymbol{\xi}_{*},
\end{aligned}
$$

where $r \in[0,1]$ and $R \in[0,1]$ are parameters, introduced in Section 3, describing the exchange of internal energy during molecular collision and $\boldsymbol{\omega} \in S^{2}$ is a unit sphere vector. As in classical case, the model of interaction between molecules is reflected on collision cross section $\mathcal{B}$. Here, we shall assume the following form of the cross section:

$$
\mathcal{B}=R^{1 / 2}\left|\xi-\xi_{*}\right|\left|\omega \cdot \frac{\xi-\xi_{*}}{\left|\xi-\xi_{*}\right|}\right|
$$

which resembles the variable hard spheres model. Furthermore, we shall assume that state of the gas during processes is not far from local equilibrium. Therefore, products of non-equilibrium distribution functions which appear in collision integral will be linearized with respect to moments of distribution functions, i.e. stress tensor $p_{i j}$ and heat flux $q_{i}$.

Statement 6. Up to first order terms in densities, production term in second order moments equation in F-hierarchy reads:

$$
P_{i j}=-\frac{4}{15} \frac{m \pi}{q^{2}(T)} \frac{p^{2}}{\rho}\left\{p_{\langle i j\rangle}+\frac{4}{7}\left(\frac{5}{2}+\alpha\right)(1+\alpha)^{-1} \Pi \delta_{i j}\right\}
$$

whereas production term in third order moments equation in G-hierarchy has the following form:

$$
\begin{aligned}
Q_{i}=- & \frac{8}{15} \frac{m \pi}{q^{2}(T)} \frac{p^{2}}{\rho}\left\{\left(p_{\langle i j\rangle}+\frac{4}{7}\left(\frac{5}{2}+\alpha\right)(1+\alpha)^{-1} \Pi \delta_{i j}\right) u_{j}\right. \\
& \left.+\frac{10}{7}\left(\frac{7}{2}+\alpha\right)^{-1} q_{i}\right\},
\end{aligned}
$$

where $p_{\langle i j\rangle}$ denotes traceless part of the pressure tensor defined by (33).

The derivation of (48) and (49) rests upon assumptions stated above and standard calculations used in kinetic theory of gases for derivation of 
production terms. The interested reader may grasp the details from the book [11], or from the Annexe 1 of [24]. These results complete the calculations needed for transfer equations for moments, i.e. 14 moments macroscopic field equations.

The results obtained here are a consequence of the model of interaction between the molecules. The production terms (48) and (49) thus represent the macroscopic dissipative effects. However, their structure reflects the mesoscopic mechanism of interaction without any prior recourse to phenomenological relations.

\subsection{The 14 moments equations and transport coefficients}

In preceding paragraphs all the necessary ingredients of the field equations are determined in 14 moments approximation. Hence, we are in position to write down the closed set of 14 moments equations. At the same time, statement that maximization of entropy is equivalent to extended thermodynamics of moments [7], calls for comparison of the results given here with ones derived in [13], obtained by the formalism of extended thermodynamics. Although the latter ones are valid for any real gas, it can be proved that they are equivalent in the case of rarefied polyatomic gases. In this way the equivalence of MEP and ET will be extended from monatomic gases [7, 10] to polyatomic ones.

In the first step, the explicit form of 14 moments equations will be given. Equations of $F$-hierarchy have the form:

$$
\begin{aligned}
& \partial_{t} \rho+\partial_{i}\left(\rho u_{i}\right)=0 \\
& \partial_{t}\left(\rho u_{i}\right)+\partial_{j}\left(\rho u_{i} u_{j}+p_{i j}\right)=0, \\
& \partial_{t}\left(\rho u_{i} u_{j}+p_{i j}\right)+\partial_{k}\left\{\rho u_{i} u_{j} u_{k}+u_{i} p_{j k}+u_{j} p_{k i}+u_{k} p_{i j}\right. \\
& \left.\quad+(7 / 2+\alpha)^{-1}\left(q_{i} \delta_{j k}+q_{j} \delta_{k i}+q_{k} \delta_{i j}\right)\right\} \\
& \quad=-\frac{4}{15} \frac{m \pi}{q^{2}(T)} \frac{p^{2}}{\rho}\left\{p_{\langle i j\rangle}+\frac{4}{7}\left(\frac{5}{2}+\alpha\right)(1+\alpha)^{-1} \Pi \delta_{i j}\right\},
\end{aligned}
$$


while equations of $G$-hierarchy read:

$$
\begin{aligned}
& \partial_{t}\left(\frac{1}{2} \rho|\mathbf{u}|^{2}+\rho \varepsilon\right)+\partial_{i}\left\{\left(\frac{1}{2} \rho|\mathbf{u}|^{2}+\rho \varepsilon\right) u_{i}+p_{i j} u_{j}+q_{i}\right\}=0, \\
& \partial_{t}\left\{\left(\frac{1}{2} \rho|\mathbf{u}|^{2}+\rho \varepsilon\right) u_{i}+p_{i j} u_{j}+q_{i}\right\}+\partial_{j}\left\{\left(\frac{1}{2} \rho|\mathbf{u}|^{2}+\rho \varepsilon\right) u_{i} u_{j}\right. \\
& \left.+u_{i} u_{k} p_{j k}+u_{j} u_{k} p_{i k}+\frac{1}{2} \rho|\mathbf{u}|^{2} p_{i j}+\left(\frac{9}{2}+\alpha\right) \frac{p}{\rho} p_{i j}-\frac{p^{2}}{\rho} \delta_{i j}\right\} \\
& =-\frac{4}{15} \frac{m \pi}{q^{2}(T)} \frac{p^{2}}{\rho}\left\{\left(p_{\langle i j\rangle}+\frac{4}{7}\left(\frac{5}{2}+\alpha\right)(1+\alpha)^{-1} \Pi \delta_{i j}\right) u_{j}\right. \\
& \left.\quad+\frac{10}{7}\left(\frac{7}{2}+\alpha\right)^{-1} q_{i}\right\}
\end{aligned}
$$

In derivation of (50)-(51) we started from (35). The internal energy $\varepsilon$ is determined by (28) and $q(T)$ by (29). We also exploited expressions for nonconvective fluxes (46)-(47) and slightly transformed version of production terms (48)-(49).

To prove the equivalence of the system (50)-(51) with equations of ET, one must take into account (31), (32), (33), use the material derivative ()$^{\circ}=$ $\partial_{t}()+u_{j} \partial_{j}()$, and exploit the relation (26) between $\alpha$ and $D$. In such a way, the system (50)-(51) is transformed into Eq. (67), given in [13], which represent 14 moments system of ET for dense gases in rarefied gas limit. Moreover, relaxation times $\tau_{S}$ for viscous stress, $\tau_{\Pi}$ for dynamic pressure and $\tau_{q}$ for heat flux, of the ET model can be explicitly calculated from our production terms:

$$
\begin{aligned}
\frac{1}{\tau_{S}} & =\frac{4}{15} \frac{m \pi}{q^{2}(T)} \frac{p^{2}}{\rho}, \\
\frac{1}{\tau_{\Pi}} & =\frac{16}{105}\left(\frac{5}{2}+\alpha\right)(1+\alpha)^{-1} \frac{m \pi}{q^{2}(T)} \frac{p^{2}}{\rho}, \\
\frac{1}{\tau_{q}} & =\frac{8}{21}\left(\frac{7}{2}+\alpha\right)^{-1} \frac{m \pi}{q^{2}(T)} \frac{p^{2}}{\rho} .
\end{aligned}
$$

In [13] it was also proved, by means of so-called Maxwellian iteration, that the classical equations of Navier-Stokes and Fourier appear as limit cases of the balance laws $(50)_{3}$ and $(51)_{2}$. Taking into account these results one 
may obtain explicit form of transport coefficients, i.e. shear viscosity $\mu$, bulk viscosity $\nu$ and heat conductivity $\kappa$ :

$$
\begin{aligned}
& \mu=p \tau_{S}=\frac{15}{4} \frac{\Gamma^{2}(1+\alpha)}{\pi}(k T)^{1+2 \alpha}, \\
& \nu=\frac{2(D-3)}{3 D} p \tau_{\Pi}=\frac{35}{8}(1+\alpha)^{2}\left(\frac{5}{2}+\alpha\right)^{-2} \frac{\Gamma^{2}(1+\alpha)}{\pi}(k T)^{1+2 \alpha}, \\
& \kappa=\frac{D+2}{2} \frac{p^{2}}{\rho T} \tau_{q}=\frac{21}{8}\left(\frac{7}{2}+\alpha\right)^{2} \frac{k}{m} \frac{\Gamma^{2}(1+\alpha)}{\pi}(k T)^{1+2 \alpha} .
\end{aligned}
$$

Note that the transport coefficients can be evaluated explicitly from the model with internal energy, in contrast to ET where only the relation to the relaxation times can be determined.

\subsection{Entropy and entropy flux}

Another important feature which we need to discuss is the structure of entropy density and entropy flux for non-equilibrium distribution (39). Macroscopic entropy is defined by (15):

$$
h=\rho s=-k \int_{\mathbb{R}^{3}} \int_{0}^{\infty} f \log f \varphi(I) d I d \boldsymbol{\xi},
$$

while entropy flux has the form:

$$
h_{j}=\rho s u_{j}+\varphi_{j}, \quad \varphi_{j}=-k \int_{\mathbb{R}^{3}} \int_{0}^{\infty} C_{j} f \log f \varphi(I) d I d \boldsymbol{\xi},
$$

where $\varphi_{j}$ is a non-convective entropy flux. As a first step, we shall derive them for the equilibrium distribution (20).

Statement 7. The entropy density and the non-convective entropy flux for polyatomic gases in local equilibrium state have the following form:

$$
\rho s_{E}=-k \frac{\rho}{m}\left\{\log \left(\frac{\rho}{m q(T)}\left(\frac{m}{2 \pi k T}\right)^{3 / 2}\right)-\left(\frac{5}{2}+\alpha\right)\right\}, \quad \varphi_{j E}=0 .
$$

Moreover, Gibbs relation holds in equilibrium:

$$
d s_{E}=\frac{1}{T}\left\{d \varepsilon_{E}-\frac{p}{\rho^{2}} d \rho\right\},
$$

where $\varepsilon_{E}$ is the internal energy density of polyatomic gas (28). 
For both relations (55) and (56) the proof is straightforward. They are derived by direct insertion of equilibrium distribution (20) into (53) and (54). Gibbs relation comes out after differentiation of $(55)_{1}$.

We can conclude from the last Statement that equilibrium entropy density presents generalization of the one for monatomic gases and the form of Gibbs relation is also preserved. The form of non-equilibrium entropy density and entropy flux is dictated by the form of non-equilibrium velocity distribution function and thus can be expressed in terms of 14 scalar fields.

Statement 8. The non-equilibrium entropy density and entropy flux for polyatomic gases in 14 moments approximation have the following form:

$$
\begin{aligned}
\rho s= & \rho s_{E}-\frac{1}{4} \frac{k}{m} \frac{\rho}{p^{2}} p_{\langle i j\rangle} p_{\langle i j\rangle}-\frac{1}{2}\left(\frac{7}{2}+\alpha\right)^{-1} \frac{k}{m} \frac{\rho^{2}}{p^{3}} q_{i} q_{i} \\
& -\frac{3}{4}\left(\frac{5}{2}+\alpha\right)(1+\alpha)^{-1} \frac{k}{m} \frac{\rho}{p^{2}} \Pi^{2}, \\
\varphi_{i}= & \frac{q_{i}}{T}-\frac{k}{m} \frac{\rho}{p^{2}}\left(\frac{7}{2}+\alpha\right)^{-1} p_{\langle i j\rangle} q_{j}-\frac{k}{m} \frac{\rho}{p^{2}}\left(\frac{7}{2}+\alpha\right)^{-1} \Pi q_{i} .
\end{aligned}
$$

To prove these results it is sufficient to follow the procedure used in the derivation of entropy density and entropy flux in the case of Grad's distribution function $[4,11]$. First, one has to put non-equilibrium distribution function (39) into (53) and (54). Since we shall restrict our study to second order terms with respect to non-equilibrium densities - pressure tensor $p_{i j}$, heat flux $q_{i}$ and dynamic pressure $\Pi$ - we shall exploit the approximation $\log (1+x) \approx x-x^{2} / 2$, valid for $|x| \ll 1$. Under these assumptions, relations (57)-(58) are obtained after tedious, but straightforward integration of (53) and (54).

Non-equilibrium entropy density (57) comprises the equilibrium one $\rho s_{E}$ and non-linear, quadratic terms related to pressure tensor, heat flux and dynamic pressure. It is a generalization of Grad's entropy density since it contains the polyatomic parameter $\alpha$ and term $\Pi^{2}$ which does not exist in monatomic case. Moreover, it fulfills the convexity conditions provided $\alpha>-1$ (or equivalently $D>3$ ), ensuring that 14 moments system (50)-(51) can be put into symmetric hyperbolic form [18].

The entropy flux (58) share the same properties as the entropy density. It contains the linear term, $q_{i} / T$, typical for linear theory, but also comprises non-linear terms $p_{\langle i j\rangle} q_{j}$, also present in monatomic case, and $\Pi q_{i}$ which is 
the non-linear contribution peculiar for polyatomic gases. The parameter $\alpha$ is also present. Also, these results are in perfect agreement with the ones of ET [13].

\subsection{Qualitative Analysis}

As we proved the equivalence between ET and MEP for rarefied polyatomic gas, we can apply the same results concerning hyperbolic system endowed with a supplementary convex entropy. According with the general results due to Godunov [23], Boillat [26] and Ruggeri \& Strumia [27], the original system can be put in symmetric form using the main field $\mathbf{u}^{\prime}$, which coincide with the Lagrange multipliers of balance laws [10]. Therefore, we can conclude that the system (50), (51) is symmetric hyperbolic (at least in neighborhood of an equilibrium state) in the main field components given by (42). Symmetric structure of the system ensures local well posedness for Cauchy problems. Moreover, it can be shown, proceeding as in [28], that the Kawashima-Shizuta condition [29] holds also for polyatomic rarefied gas. Hence, all the previous general results [30, 31, 32, 33] hold and global smooth solutions of the system exist, provided initial data are sufficiently small.

\section{Conclusions}

In this paper we applied maximum entropy principle to the model of polyatomic rarefied gases with continuous internal energy. The aim was to derive approximate non-equilibrium velocity distribution function in the spirit of Grad's moment method, and to exploit it in closure procedure for transfer equations for moments. Since there was no simple relation between the trace of pressure tensor and internal energy density, like the one for monatomic gases, it was needed to establish two independent hierarchies of moment equations - the momentum and the energy one. This is the first novelty in our study.

By the application of maximum entropy principle, we first recovered the equilibrium distribution function (20), as well as Euler gas dynamics equations. In the next step the approximate non-equilibrium distribution function (39) was derived. Solution of the variational problem with constraints comprises 14 moments - mass and momentum density, pressure tensor, energy density and heat flux - and naturally proves the existence of dynamic pressure. This solution was used to resolve the closure problem for the system of 14 moment equations (35). The non-convective fluxes (46)-(47) for the 
highest order moment equations were derived, as well as production terms (48)-(49). With all these ingredients we were able to close the system of field equations (50)-(51) and to determine the transport coefficients (52). Finally, entropy density and entropy flux (53)-(54) were calculated for equilibrium distribution and for non-equilibrium one (57)-(58) and the Gibbs (56) relation was proved in local equilibrium state. As far as the authors are concerned, the results listed above are derived for the first time, along with idea to use two hierarchies of moment equations instead of one.

The results presented in this paper can be regarded just as the tip of the iceberg. Due to equivalence of maximum entropy principle and extended thermodynamics of moments, further study of polyatomic gases can be based upon either standpoint. At this moment, two classes of problems may be roughly depicted. One is related to the general structure of hierarchies of moment equations. Nested structure of classical hierarchy was thoroughly analyzed in [18]. Thus, there appears the question about the level of truncation in each hierarchy, which is important for both formal mathematical reasons and physical interpretation. Another path of study is paved with particular flow problems (like linear and non-linear waves, shock structure etc.) and analysis of transport coefficients for different models of interaction, i.e. different choices of collision cross-section $\mathcal{B}$. Furthermore, following [21] one may proceed in a similar way with the mixture modelling. These are some hints about possible problems which could become the part of a bigger project related to the modelling of real gases.

Acknowledgement. This work was supported partially (Milana Pavić and Srboljub Simić) by the Ministry of Education and Science, Republic of Serbia, through the project "Mechanics of nonlinear and dissipative systems contemporary models, analysis and applications", Project No. ON174016, and partially (Tommaso Ruggeri) by the National Group of Mathematical Physics, GNFM-INdAM. The author express their gratitude to Takashi Arima and Shigeru Taniguchi from Nagoya Institute of Technology, Japan, for many useful comments and help during the preparation of the paper.

[1] J.-F. Bourgat, L. Desvillettes, P. Le Tallec and B. Perthame (1994) Microreversible collisions for polyatomic gases, Eur. J. Mech., B/Fluids, 13 (2), 237-254.

[2] I. Müller and T. Ruggeri (1998) Rational Extended Thermodynamics, 
2nd ed., Springer Tracts in Natural Philosophy 37, Springer-Verlag, New York.

[3] I. Müller and W. Weiss (2012) Thermodynamics of irreversible processes - past and present, Eur. Phys. J. H, 37, 139-236.

[4] H. Grad (1949) On the Kinetic Theory of Rarefied Gases, Commun. Pure Appl. Math., 2, 331-407.

[5] M.N. Kogan (1967) On the principle of maximum entropy, in Rarefied Gas Dynamics, Vol. I, 359-368, Academic Press, New York.

[6] I-S. Liu and I. Müller (1983) Extended thermodynamics of classical and degenerate ideal gases, Arch. Rat. Mech. Anal. 83, 285-332.

[7] W. Dreyer (1987) Maximisation of the entropy in non-equilibrium, $J$. Phys. A: Math. Gen., 20, 6505-6517.

[8] I. Müller and T. Ruggeri (1993) Extended Thermodynamics, Springer Tracts in Natural Philosophy 37, Springer-Verlag, New York.

[9] C.D. Levermore (1996) Moment Closure Hierarchies for Kinetic Theories, Journal of Statistical Physics, 83 (5/6), 1021-1065.

[10] G. Boillat and T. Ruggeri (1997) Moment equations in the kinetic theory of gases and wave velocities, Continuum Mech. Thermodyn., 9, 205-212.

[11] G.M. Kremer (2010) An Introduction to the Boltzmann Equation and Transport Processes in Gases, Springer-Verlag, Berlin.

[12] C. Borgnakke and P.S. Larsen (1975) Statistical Collision Model for Monte Carlo Simulation of Polyatomic Gas Mixture, Journal of Computational Physics, 18, 405-420.

[13] T. Arima, S. Taniguchi, T. Ruggeri and M. Sugiyama (2011) Extended thermodynamics of dense gases, Continuum Mech. Thermodyn., doi: 10.1007/s00161-011-0213-x.

[14] I-S. Liu (1985) Extended thermodynamics of fuids and virial equations of state, Arch. Rational Mech. Anal., 88, 1-23. 
[15] G.M. Kremer (1987) Extended thermodynamics of non-ideal gases. Physica A, 144, 156-178.

[16] T. Arima, S. Taniguchi, T. Ruggeri and M. Sugiyama (2012) Extended thermodynamics of real gases with dynamic pressure: An extension of Meixners theory, Physics Letters A, http://dx.doi.org/10.1016/j.physleta.2012.08.030, in press.

[17] M.N. Kogan (1969) Rarefied Gas Dynamics, Plenum Press, New York.

[18] G. Boillat and T. Ruggeri (1997) Hyperbolic Principal Subsystems: Entropy Convexity and Subcharacteristic Conditions, Arch. Rat. Mech. Anal., 137, 305-320.

[19] F. Brini and T. Ruggeri (2002) Entropy principle for the moment systems of degree $\alpha$ associated to the Boltzmann equation. Critical derivatives and non controllable boundary data, Continuum Mech. Thermodyn., 14, 165-189.

[20] J-H. Schönfeldt, N. Jimenez, A.R. Plastino, A. Plastino and M. Casas (2007) Maximum entropy principle and classical evolution equations with source terms, Physica A, 374, 573-584.

[21] L. Desvillettes, R. Monaco and F. Salvarani (2005) A kinetic model allowing to obtain the energy law of polytropic gases in the presence of chemical reactions, European Journal of Mechanics B/Fluids, 24, 219236.

[22] T. Ruggeri (1989) Galilean invariance and entropy principle for systems of balance laws. The structure of extended thermodynamics, Continuum Mech. Thermodyn., 1, 3-20.

[23] S.K. Godunov (1961) An interesting class of quasilinear systems, Sov. Math. Dokl.

[24] F. Mallinger (1998) Generalization of the Grad theory to polyatomic gases, INRIA - Research Report, No. 3581.

[25] C.S. Wang Chang, G.E. Uhlenbeck and J. de Boer (1964) The heat conductivity and viscosity of plyatomic gases, in Studies in Statistical Mechanics, Vol. II, 243-268, North-Holland, Amsterdam. 
[26] G. Boillat (1974) Sur l'existence et la recherche d'équations de conservation supplémentaires pour les systémes hyperboliques, C. R. Acad Sci. Paris A 278, 909-912.

[27] T. Ruggeri, and A. Strumia (1981) Main field and convex covariant density for quasi-linear hyperbolic systems. Relativistic fluid dynamics, Ann. Inst. H. Poincaré. Section A 34, 65-84.

[28] T. Ruggeri (2005) Global existence of smooth solutions and stability of the constant state for dissipative hyperbolic systems with applications to extended thermodynamics, in Trends and Applications of Mathematics to Mechanics, Editors S. Rionero, G. Romano, 215-224, Springer, Berlin.

[29] Y. Shizuta and S. Kawashima (1985) Systems of equations of hyperbolicparabolic type with applications to the discrete Boltzmann equation, Hokkaido Math. J., 14, 249.

[30] B. Hanouzet, R.Natalini (2003) Global existence of smooth solutions for partially dissipative hyperbolic systems with a convex entropy, Arch. Rat. Mech. Anal., 169, 89.

[31] Wen An Yong (2004) Entropy and global existence for hyperbolic balance laws, Arch. Rat. Mech. Anal., 172, 247.

[32] T. Ruggeri, D. Serre (2004) Stability of constant equilibrium state for dissipative balance laws system with a convex entropy, Quart. Appl. Math, 62 (1), 163.

[33] S. Bianchini, B. Hanouzet, R.Natalini (2007) Asymptotic Behavior of Smooth Solutions for Partially Dissipative Hyperbolic Systems with a Convex Entropy, Comm. Pure Appl. Math., 60, 1559. 